\section{A Case of Idiopathic Adulthood Ductopenia}

A case of idiopathic adulthood ductopenia (IAD) (1) characterized by chronic cholestatic liver disease associated with an unexplained loss of the interlobular bile ducts is presented here. A 56-year-old man was admitted to our hospital in 1988, with liver dysfunction and esophageal varices. He had no past history of liver disease, blood transfusion, or alcohol abuse. His drug history was negative. There was no family history of liver disease. Laboratory data indicated cholestatic liver injury. Hepatitis A, B, and C serologies were negative. AMA was absent, and the serum IgM was in the normal range. An abdominal ultrasound scan and CT revealed hepatosplenomegaly. An endoscopic examination showed only esophageal varices, and colonoscopy showed no evidence of inflammatory bowel disease. On endoscopic retrograde cholangiography, the bile ducts were normal. The findings of laparoscopy and liver biopsy were compatible with IAD. He had a clinical course of chronic and

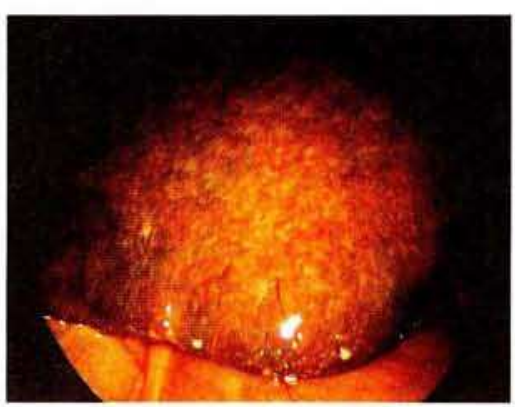

Laparoscopy shows a remarkably enlarged, green liver (especially in the left lobe) with a granular surface.

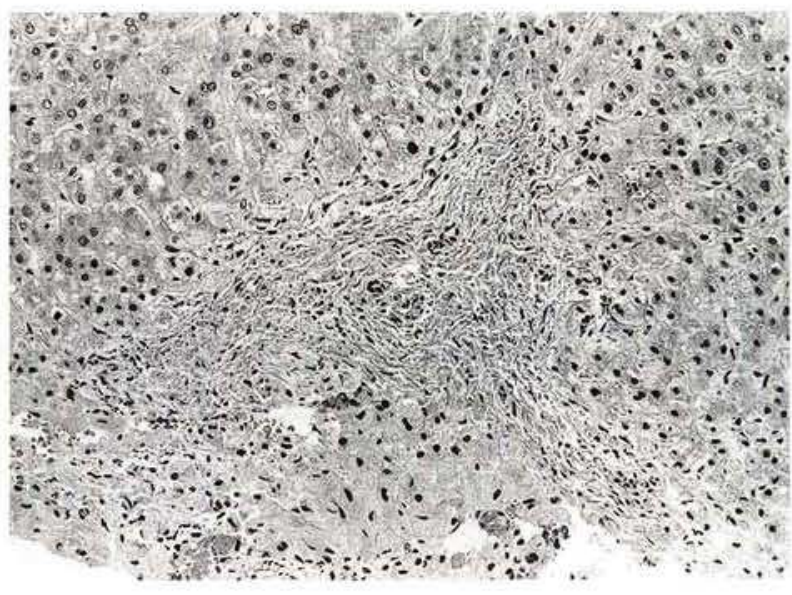

Figure 2: Histology of the liver shows minimal inflammatory cell infiltration in the portal tracts, but the fibrosis is marked and progressive. The interlobular bile ducts have degenerated, but no evidence of fibrous obliterative cholangitis or chronic nonsuppurative cholangitis is seen. progressive intrahepatic cholestasis. Ursodeoxycholic acid, steroid, and phenobarbital treatment were not effective. The findings of the third laparoscopy and liver biopsy, carried out in 1991, are shown in Figures $\mathbf{1}$ and $\mathbf{2}$. 
The histologic features of our case indicated marked destruction and degeneration of the interlobular bile ducts compatible with IAD. Primary biliary cirrhosis, primary sclerosing cholangitis, drug-induced cholestasis, and other known diseases were also excluded. IAD is a progressive disease, and the etiology and treatment are still unknown.

K. Nakajima, M. Komatsu, T. Ono, T. Kuramitsu, M. Goto, R. Ito, O. Masamune

First Department of Internal Medicine Akita University School of Medicine

Hondo 1-1-1, Akita City, Japan 010

\section{References}

1. Ludwig J, Wiesner RH, LaRusso NF: Idiopathic adulthood ductopenia: a cause of chronic cholestatic liver disease and biliary cirrhosis. J. Hepatol. 1988; 7: 193-199. 